\title{
In Vivo Assessment of Cortical Bone Fragility
}

\author{
Lyn Bowman $^{1,2} \cdot$ Anne B. Loucks ${ }^{1}$
}

Published online: 22 February 2020

(C) The Author(s) 2020

\begin{abstract}
Purpose of Review This review updates readers on recent developments in the assessment of cortical bone fragility in vivo. The review explains the clinical need that motivated the development of Cortical Bone Mechanics Technology ${ }^{\mathrm{TM}}$ (CBMT) as a scientific instrument, its unique capabilities, and its necessary further development as a medical device.

Recent Findings Clinical experience with dual-energy X-ray absorptiometry has led to calls for new clinical methods for assessing bone health. CBMT is a noninvasive, dynamic 3-point bending test that makes direct, functional measurements of the mechanical properties of cortical bone in ulnas of living people. Its technical validity in accurate measurements of ulna flexural rigidity and its clinical validity in accurate estimations of quasistatic ulna bending strength have been demonstrated. Summary Because CBMT is a whole bone test, its measurements reflect the influences of bone quantity and bone quality at all hierarchical levels.
\end{abstract}

Keywords Fracture $\cdot$ Cortical bone $\cdot$ Bone mechanics $\cdot$ Bending test $\cdot$ Bone stiffness $\cdot$ Bone strength

\section{Introduction}

More than 40 years ago, quasistatic, single load-to-failure tests of whole long bones ex vivo demonstrated that bending stiffness is a very accurate predictor of the maximum load a bone can bear before breaking $[1,2]$. Cortical Bone Mechanics Technology ${ }^{\mathrm{TM}}$ (CBMT) performs a dynamic 3-point bending test to make direct, noninvasive measurements of ulna bending stiffness in humans in vivo. Recent quasistatic validation of these measurements has confirmed that they also predict ulna bending strength very accurately $\left[3^{\bullet}\right]$.

Because CBMT is a whole bone test, its measurements reflect the sum total of all factors operating at all hierarchical levels of the ulna, from whole bone geometry to tissue material properties and composition, porosity, microarchitecture, and nanoscale collagen cross-linking and protein-mineral bonding [4]. Thus, CBMT captures the influences of bone

This article is part of the Topical Collection on Biomechanics

Anne B. Loucks

loucks@ohio.edu

1 Department of Biological Sciences and the Ohio Musculoskeletal and Neurological Institute, Ohio University, Athens, $\mathrm{OH} 45701$, USA

2 AEIOU Scientific, LLC, Ohio University, Athens, OH 45701, USA quality as well as bone quantity on the ulna's load-bearing capacity, however, that may have been affected by genetics, modeling, remodeling, nutrition, activity, aging, disease, pharmacological intervention, etc.

We describe here our motivation for developing and commercializing CBMT, its unusual capabilities, and its remaining unknowns.

\section{The Clinical Need: Fewer Errors in Allocating Patients to Treatment}

T-scores of areal bone mineral density (aBMD) measured by dual-energy X-ray absorptiometry (DXA) perform well in their original epidemiological application [5]. They identify a subpopulation of patients (those with aBMD T-scores $<$ -2.5 ) with a higher risk of fracture than the rest. It is in their subsequent clinical application for allocating individuals to treatment to prevent fractures that aBMD T-scores have disappointed expectations by failing to predict fractures well [6]. As will be shown below, most patients diagnosed with osteoporosis in epidemiological studies have not fractured, and most fractures have occurred among patients who did not have osteoporosis.

In the field of machine learning, the performance of a method for binary classification of a population can be assessed by a type of contingency table known as a confusion matrix [7]. 
Figure 1 shows the confusion matrix for binary assignment of patients to treatment by aBMD T-scores. Patients expected to fracture $(\mathrm{FX} \mathrm{T}<-2.5)$ are assigned to treatment, while treatment is withheld from those not expected to fracture (NFX T >-2.5). Actual outcomes differ. Some patients allocated to treatment do fracture ( $\mathrm{TP}=$ True Positive), but others do not ( $\mathrm{FP}=$ False Positive), and some patients from whom treatment is withheld do not fracture ( $\mathrm{TN}=$ True Negative), while others do ( $\mathrm{FN}=$ False Negative).

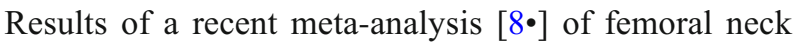
aBMD T-scores from 7254 women and men in eight studies of fractures are summarized in the confusion matrix shown in Fig. 2. For clarity numbers have been normalized to 1000 participants. The risk of fracture in those eligible for treatment $\left(\mathrm{T}_{\mathrm{aBMD}}<-2.5\right)$ was about double the risk in those who were $\operatorname{not}\left(\mathrm{T}_{\mathrm{aBMD}}>-2.5\right)$ :

$$
\begin{aligned}
\mathrm{RR} & =[\mathrm{TP} /(\mathrm{TP}+\mathrm{FP})] /[\mathrm{FN} /(\mathrm{FN}+\mathrm{TN})] \\
\mathrm{RR}_{\mathrm{Women}} & =[20 /(20+79)] /[67 /(96+823)]=1.9 \\
\mathrm{RR}_{\text {Men }} & =[4 /(4+27)] /[75 /(75+894)]=2.1
\end{aligned}
$$

In a confusion matrix, the statistics that quantify misallocations of individual patients to treatment are the false positive rate (FPR) and false negative rate (FNR). Figure 2 shows that most women eligible for treatment did not fracture (FPR $=80 \%$ ), and most fractures occurred in women who were not eligible $(F N R=83 \%)$. Results for men were even worse $(\mathrm{FPR}=86 \%, \mathrm{FNR}=95 \%)$.

$$
\begin{aligned}
\mathrm{FPR} & =\mathrm{FP} /(\mathrm{FP}+\mathrm{TP}) \\
\mathrm{FNR} & =\mathrm{FN} /(\mathrm{FN}+\mathrm{TP}) \\
\mathrm{FPR}_{\mathrm{Women}} & =100[79 /(79+20)]=80 \% \\
\mathrm{FNR}_{\text {Women }} & =100[96 /(96+20)]=83 \% \\
\mathrm{FPR}_{\text {Men }} & =100[27 /(27+4)]=86 \% \\
\mathrm{FNR}_{\text {Men }} & =100[75 /(75+4)]=95 \%
\end{aligned}
$$

These high FNR observations seem inexplicable by anything other than misallocation of treatment. However, might the high FPR observations be the happy results of effective fracture preventive care correctly targeted at the patients who need it most? Unfortunately, it would seem not. Of the eight studies included in Fig. 2, two (MrOS in Sweden and Strambo in France) observed only men. Focusing on the high FPR in women in the other six studies, one (Qualyor in France) excluded participants taking osteoporosis medications. Investigators in two of the other five studies responded to our inquiries with information on the use of osteoporosis medications by their participants. In the CaMOS study in Canada, $39 \%$ of participants with aBMD T-scores $<-2.5$ had taken osteoporosis medications [9], and in the GERICO study in

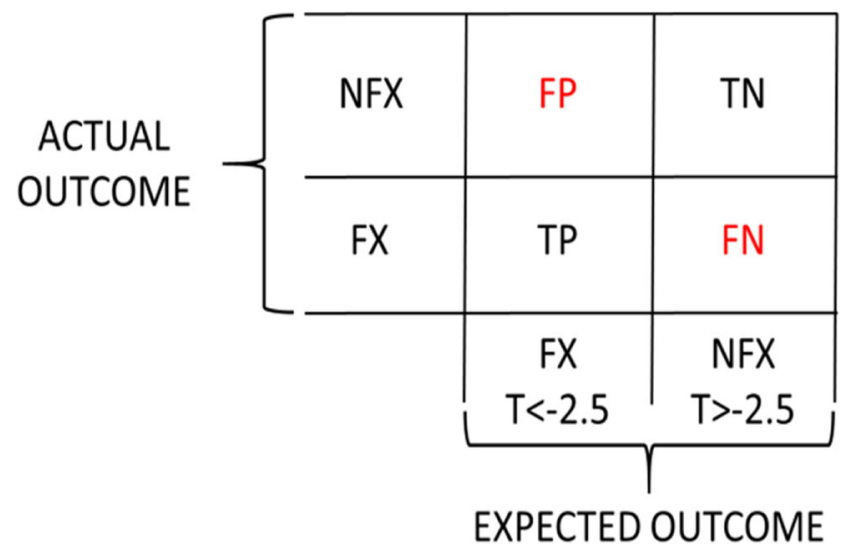

Fig. 1 The confusion matrix for using aBMD T-scores for allocating patients to treatment to prevent fractures. $\mathrm{TP}=$ True Positive, $\mathrm{FP}=$ False Positive, $\mathrm{FN}=$ False Negative, $\mathrm{TN}=$ True Negative

Switzerland, only $19 \%$ of such participants had done so [10]. In the latter study, most of the osteoporosis medication was in the form of menopausal hormonal therapy taken for reasons other than skeletal health, as it was by similar proportions of participants in osteopenic and normal ranges of aBMD T-scores. Therefore, information from three of the six studies of women indicates that the use of osteoporosis medication does not account for the high FPR in women diagnosed with osteoporosis. And so, we are thrown back on
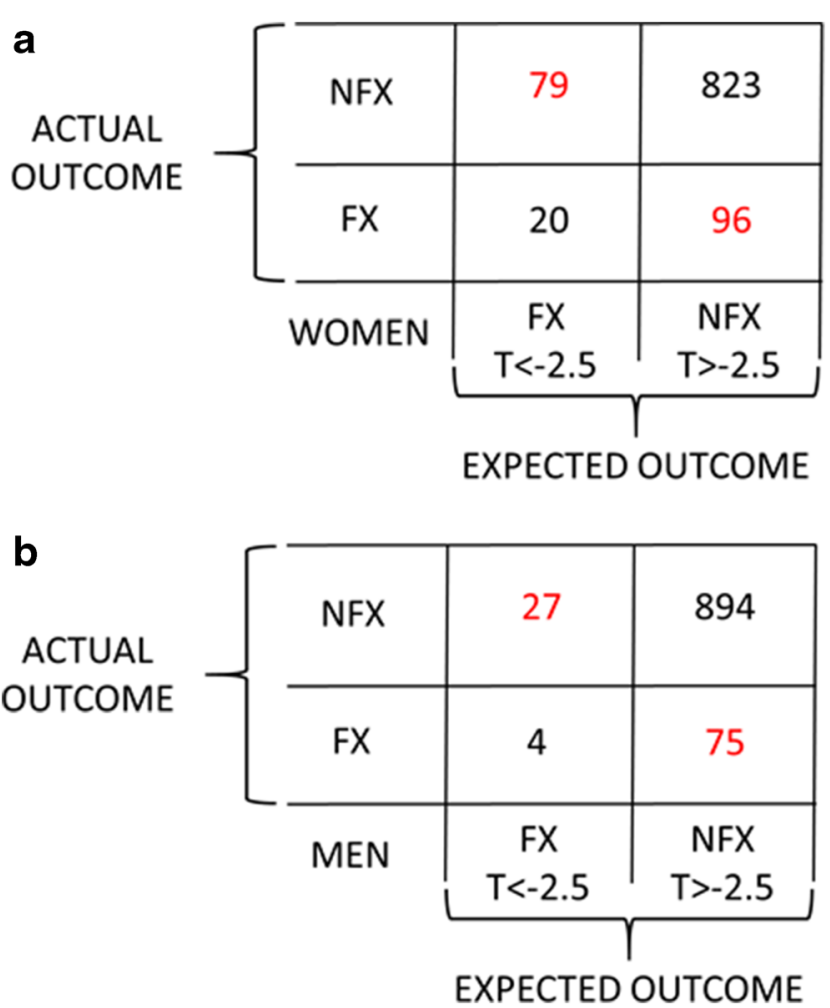

Fig. 2 The confusion matrix for allocations of 1000 women (a) and 1000 men (b) to treatment based on T-scores of aBMD. FX $=$ fracture, NFX= no fracture, $\mathrm{FX} \mathrm{T}<-2.5=$ fracture expected, NFX T $>-2.5=$ fracture not expected. (Data from [8•]) 
misallocation of treatment as the most likely explanation for the high FPR as well as the high FNR values observed.

FPR and FNR are important because they are the major drivers of fracture health-care costs. FP (Type 1) errors drive up cost by administering preventive care to patients who do not need it; and FN (Type 2) errors drive up cost by withholding preventive care from patients who do need it and then require treatment for fracture repair. Increasing attention to these disappointing observations $[11,12]$ has led to widening agreement that "new methods for assessing bone health are required, beyond characterization of mineral density" $[4,13]$. We suggest that FPR and FNR be the metrics by which such new methods are judged.

\section{What to Do?}

A fracture is a mechanical failure of a bone, and the strength of a bone is the maximum load that the bone can bear before breaking. A bone can be weakened prior to fracture by the accumulation of microdamage through cyclic fatigue [14], but still the load that breaks a fatigued bone is its strength in its weakened state.

A caveat is warranted before proceeding. A bone does not have a single strength: the maximum load that a bone can bear depends on whether it is loaded in tension, compression, torsion, shear, bending, or in some combination of these modes. Moreover, because long bones are not axisymmetric, their strength in bending and shear depends upon the direction in which they are loaded, e.g., antero-posteriorly or medio-laterally. As will be shown below, the strength of a bone also depends strongly on whether it is loaded quasistatically (i.e., very, very slowly) as in the widely accepted mechanical single load-to-failure test or dynamically (i.e., rapidly) as in the falls and collisions that occur in real life.

That said, what explains the observations summarized in Fig. 2? The answer to this question is important, because it guides strategy for efforts to improve observations of future clinical outcomes. Two contending hypotheses have been offered.

\section{The Weak Bone Hypothesis}

The Weak Bone Hypothesis holds that people suffer fractures because their bones are weak. By this hypothesis, Fig. 2 infers that aBMD T-scores must not estimate bone strength accurately. This leads to the perceived need for a new clinical method for estimating bone strength more accurately, so that treatment can be targeted at the correct patients: i.e., at FN instead of FP.

Measuring bone strength directly is not a clinical option, because a bone must be broken in order to learn how strong it was before it broke. So, for treatment decisions, an indirect method is required to measure something else that is correlated with bone strength. This predicament is illustrated in Fig. 3 in which one (or conceivably some combination) of the many bone strengths that might be measured appears on the Y-axis, and one (or some combination) of the many correlated predictors of bone strength appears on the $\mathrm{X}$-axis [15].

If the Weak Bone Hypothesis is true, treatment should be administered to patients who fall below some threshold value of $Y, Y_{\text {Treat }}$ Instead, treatment is administered to patients who fall below the associated threshold value of $\mathrm{X}, \mathrm{X}_{\text {Treat }}$. At present, $\mathrm{X}_{\text {Treat }}=\mathrm{T}_{\mathrm{aBMD}}=-2.5$.

In Fig. 3, the relationship between $\mathrm{Y}$ and $\mathrm{X}$ is described by the diagonal regression line $\hat{Y}=m X+b$, where $m$ is the slope and $\mathrm{b}$ is the Y-intercept of the regression line, and by the standard error of the estimate, SEE, which quantifies the scatter of data points from individual patients around the regression line. Because one of the assumptions of regression analysis is that data points are dispersed uniformly across the entire range of $X$, the bounds of $95 \%$ of the population are represented in Fig. 3 by a pair of lines parallel to the regression line and separated from it by $\pm Z_{95} \times$ SEE, where $Z$ is the standardized normal variable and $Z_{95}=1.96$. (Note that these parallel bounds of $95 \%$ of the population are not the curved bounds of the confidence intervals on the location of the regression line.) The vertical height of the data cloud $\left( \pm Z_{95} \times\right.$ SEE) is determined by all the systematic and random sources of error inherent in using $\mathrm{X}$ to estimate Y. For aBMD T-scores, the systematic sources of error include everything DXA does not measure, i.e., bone quality.

In Fig. 3, the binary horizontal and vertical treatment decision lines intersecting at the point $\left(\mathrm{X}_{\text {Treat }}, \mathrm{Y}_{\text {Treat }}\right)$ and the diagonal bounds of the population discriminate between the True and False Positive and the True and False Negative measures of bone strength and allocations of patients to treatment. Notice that the quadrant form of Fig. 3 corresponds to that of Figs. 1 and 2.

Through elementary geometry and algebra, one can quickly derive from Fig. 3 that for $95 \%$ of the population, the total error rate $[\mathrm{TER}=(\mathrm{FP}+\mathrm{FN}) / \mathrm{ALL}]$ in allocating patients to treatment is:

$$
\mathrm{TER}=\frac{Z_{95} S E E}{2\left(\hat{Y}_{\max }-\hat{Y}_{\text {min }}\right)} \approx \frac{S E E}{\hat{Y}_{\max }-\hat{Y}_{\text {min }}}
$$

TER can be readily calculated by visual inspection of graphs in publications of the mechanical validation of various methods. We will return to this below. For now, notice one last thing about Fig. 3: consistent with the Weak Bone Hypothesis, the strength $(\mathrm{Y})$ of FN is less than that of FP.

Others have explained the observations in Fig. 2 by arguing that treatment decisions for fracture prevention have been based on the wrong property of the wrong type of bone at the wrong skeletal sites [16]. Since most fractures occur at non-vertebral, non-hip sites of predominantly cortical bone in the appendicular skeleton, aBMD T-scores at sites of 
predominantly trabecular bone in the axial skeleton cannot be expected to predict fractures well. Moreover, since cortical porosity increases with age, treatment decisions need to be guided by a measure of cortical porosity. In addition, since the resistance to bending of cortical bone in the long bones of the appendicular skeleton increases with the fourth power of their diameter, a method is needed for assessing the crosssectional size of cortical bone. It will be noticed that these criticisms, as well as those pertaining to other neglected aspects of bone quality, all presume the Weak Bone Hypothesis and bear only upon the accuracy with which bone strength is assessed.

Despite the intuitive appeal of the Weak Bone Hypothesis, there is a plausible reason to doubt it, and that reason is classic in the history of science: The Weak Bone Hypothesis fails the parsimony test. The observations in Fig. 2 can be explained without it.

\section{The Heavy Load Hypothesis}

The Heavy Load Hypothesis holds that people who suffer fractures are exposed to excessively heavy loads. Assuming the subpopulations of women who did and did not fracture in the studies summarized in Fig. 2 (left) were normally distributed on aBMD T-scores, these distributions can be reconstructed from the reported proportions of women with osteoporotic $(\mathrm{T}<-2.5)$, osteopenic $(-2.5<\mathrm{T}<-1)$, and normal $(\mathrm{T}>-1) \mathrm{T}$-scores in the studies. In Fig. 4, the proportion of women with $\mathrm{T}_{\mathrm{aBMD}}<-2.5$ who fractured is $\mathrm{RR}=1.9$ times the proportion of women with $\mathrm{T}_{\mathrm{aBMD}}>-2.5$, as in Fig. 2. In Fig. 4, too, $80 \%$ of women allocated to treatment are not fractured ( $\mathrm{FP}$ in $\mathrm{T}_{\mathrm{aBMD}}<-2.5$ ), and $83 \%$ of fractures are in women who did not qualify for treatment ( $\mathrm{FN}$ in $\mathrm{T}_{\mathrm{aBMD}}<$ -2.5). Clearly, these high error rates occur because the distributions of FX and NFX overlap broadly across the range of aBMD T-scores.

Of course, regardless of how much two distributions overlap, the Central Limit Theorem states that in studies of groups, there is a sample size large enough to enable a difference in $\mathrm{T}_{\mathrm{aBMD}}$ to be detected between FX and NFX through statistical hypothesis testing. The Central Limit Theorem does not apply to observations of individuals, however, and so it does not help physicians make treatment decisions for individual patients.

Now suppose the women in Fig. 2 come from a single normal distribution of bone strength instead of aBMD Tscores (All in Fig. 5 below); and suppose the distribution of forces acting upon them every day is skewed, with small forces outnumbering large ones (Forces in Fig. 5). Then multiplying the two distributions generates the same FX and NFX distributions as those in Fig. 4! Moreover, in Fig. 5, FN do not have undetected weak bone, as in Fig. 3. FN have strong bone and are numerous in Fig. 5 only because of the law of large numbers: the large number of women above the diagnostic threshold of bone strength overwhelms the rarity of the large forces that act upon them.

Thus, the Heavy Load Hypothesis infers from Fig. 5 that FN in Fig. 2 did not fracture because they had weak bones. Rather, they fell or were otherwise impacted by large forces [17]. If the Heavy Load Hypothesis is true, a more effective strategy for reducing FPR and FNR would be to identify patients with poor neuro-motor function and to treat them to prevent falls [18]: dance class and table tennis, perhaps, instead of calcium and bisphosphonates. Moreover, if the Heavy
Fig. 3 Errors in the treatment of patients below a threshold value $\left(\mathrm{X}_{\text {Treat }}\right)$ of a bone strength (Y) predictor $\mathrm{X}$. ( $\mathrm{TP}=$ True Positive, $\mathrm{FP}=$ False Positive, $\mathrm{FN}=$ False Negative, $\mathrm{TN}=$ True Negative)

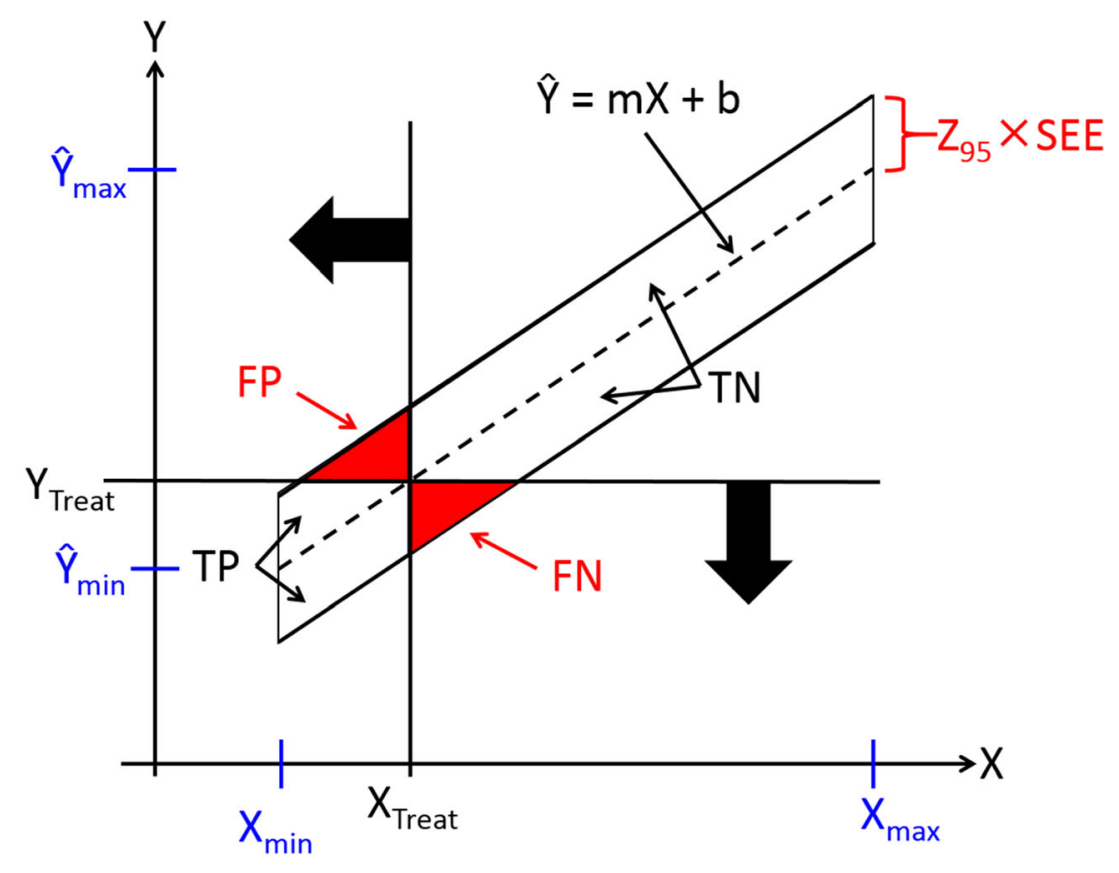


Fig. 4 Observed distributions of women with and without fractures threshold $\mathrm{T}_{\mathrm{aBMD}}=-2.5$. $(\mathrm{FX}=$ women with fractures, NFX = women without fractures, $\mathrm{TP}=$ True Positive, FP = False $\mathrm{TN}=$ True Negative, $\mathrm{dT}=$ increment of T-score) allocated to treatment by the Positive, $\mathrm{FN}=$ False Negative,

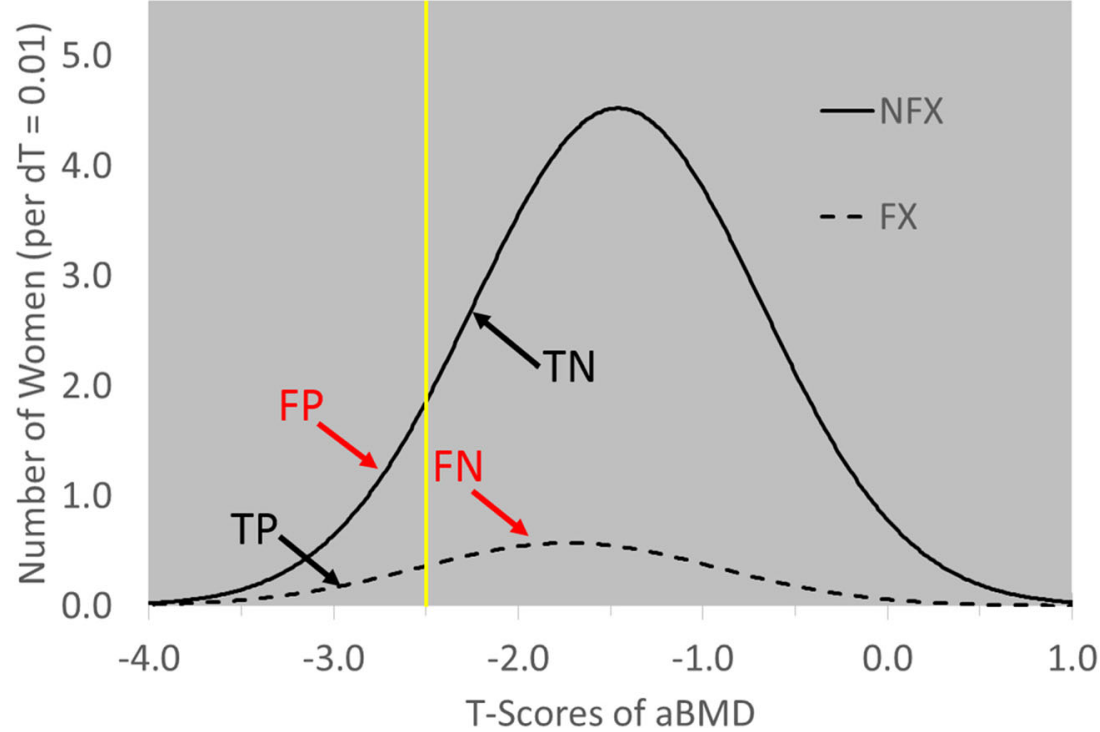

Cortical Bone Mechanics Technology ${ }^{\mathrm{TM}}$ (CBMT)

Load Hypothesis is true, then treatment of patients with more accurately estimated weak bone would not improve FPR and $F N R$, because FP would continue to be treated and FN would continue to go untreated.

If an accurate method for measuring bone strength was to emerge; however, one of the first things to do with it would be to test the Weak Bone and Heavy Load Hypotheses. In science, hypotheses are tested by falsifying them. Accordingly, if patients were to be allocated to treatment by $\mathrm{T}_{\mathrm{aBMD}}<-2.5$ and treatment were to be withheld from those with $\mathrm{T}_{\mathrm{aBMD}}>-2.5$ as in current practice and:

- If the new, more accurate method were to find bone in FN to be stronger than bone in FP, then the Weak Bone Hypothesis would be false.

- If the new, more accurate method were to find bone in FN to be weaker than bone in FP, then the Heavy Load Hypothesis would be false.
Unlike the monotonically increasing displacement imposed directly onto a bone in ex vivo quasistatic single load-to-failure tests, CBMT imposes only a small static force (10-20 N=2-4 pounds) and an even smaller oscillatory force $(1 N=3 \mathrm{oz})$ to the skin overlying the mid-shaft of the ulna bone. The static load is like the force felt on a fingertip when pressing an elevator button, and the oscillatory force is like the vibration one feels when holding an electric toothbrush. The oscillations range in frequency from 40 to $1200 \mathrm{~Hz}$.

A CBMT test is a functional test in that it measures how the ulna actually behaves mechanically (i.e., bends) under an applied load. Figure 6 shows a CBMT test in progress. The operator positions the subject for testing and then starts a robot that lowers and lifts the probe, collects and analyzes data under controlled loading conditions at various sites across the
Fig. 5 Hypothetical distributions of strength in women with and without fractures exposed to a skewed distribution of forces. (All: white solid line $=$ all women; NFX: black solid line $=$ women with fractures, FX: black broken line $=$ women without fractures; Forces: blue solid line; treatment threshold: yellow solid line at $\mathrm{T}_{\text {Strength }}=-2.5 ; \mathrm{TP}=$ True Positive, FP = False Positive, $\mathrm{FN}=$ False Negative, $\mathrm{TN}=$ True Negative)

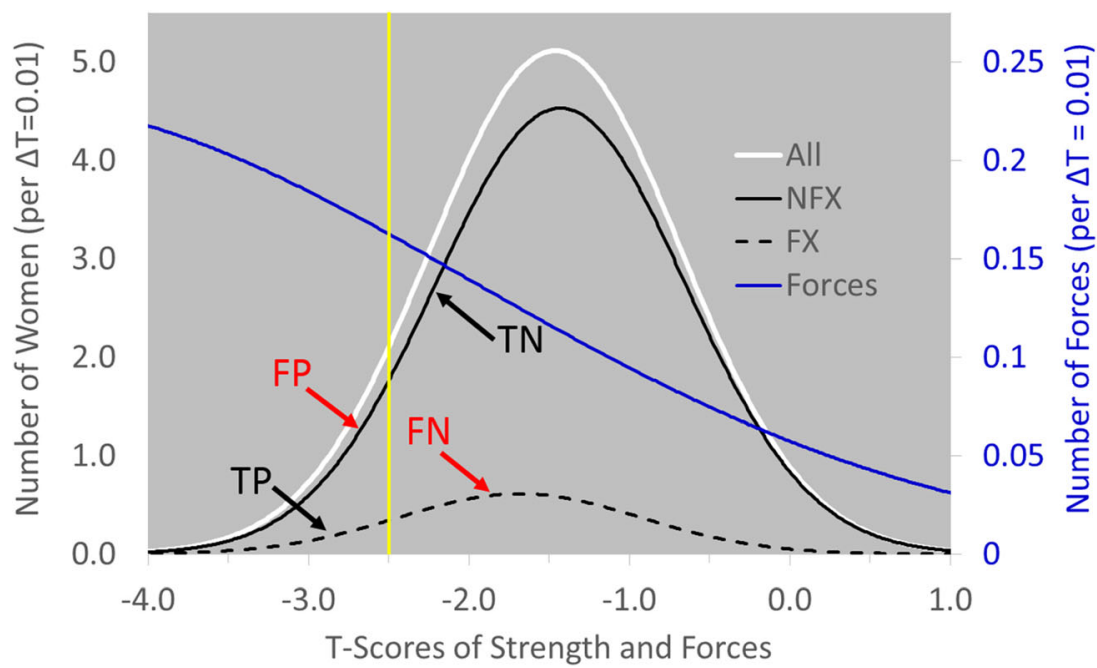


ulna, and reports results immediately. The test takes $15-$ $20 \mathrm{~min}$ to complete.

The ulna is tested because, of all the bones in the body, the biomechanics of the ulna are the most ideal for a bending test in vivo. As shown in the close-up view in Fig. 6, the ulna can be positioned with its proximal end supported at the trochlear notch by the articulating trochlea of the humerus and with its distal end supported by a rigid platform via the styloid process of the radius. No other long bone in the body can be so supported. A bending test is performed to ensure that the measured bone tissue is unambiguously cortical: a bending test is especially sensitive to mechanical properties at the mid-shaft of a long bone, where bone tissue is entirely cortical. Any apparently trabecular bone at the mid-shaft of long bones is actually "trabecularized" residual cortical bone between intracortical resorption spaces. By contrast, the distinction between cortical and trabecular bone at the distal ends of long bones is ambiguous [19].

Force and acceleration data are analyzed as a frequency response function that is fitted to a 7-parameter mathematical model of the mechanical skin-bone system to quantify the stiffness, damping and mass of the ulna and its overlying skin, as well as the damping of the surrounding soft tissue that the bone pushes aside as it vibrates. Because comparisons of ulna bending stiffness $(\mathrm{k})$ are confounded by differences in ulna length (L), ulna bending stiffness is converted to ulna flexural rigidity $\left(E I=\mathrm{kL}^{3} / 48\right)$ by Euler-Bernoulli beam theory [20] to enable ulnas of different people to be compared to one another. EI is the product of the elastic modulus (E) of ulna bone material and the cross-sectional moment of inertia (I) of ulna bone geometry in antero-posterior bending. It is to be emphasized that EI is thereby derived as a product without separate determination of $E$ or I. Thus, the values of $E$ and I implicit in EI are both patient-specific rather than assumed a priori or derived from imagery of bone mineral.

In the above calculations, CBMT is identical to a previous method known as Mechanical Response Tissue Analysis (MRTA) [20]. MRTA was developed by Charles Steele at Stanford University in the 1980s, patented by Stanford, and licensed for commercialization. Commercialization was abandoned in the 1990s when the adoption of DXA for diagnosing osteoporosis eliminated the incentive and investment available for further development. Recently, Ohio University patented novel methods described elsewhere [3•,21, 22•] for correcting sources of error in MRTA caused by its high sensitivity to probe positioning and by the impossibility of an operator identifying the correct place to position the probe by sight or touch. That problem is overcome by artificial intelligence in the CBMT robot derived from extensive studies of cadaveric human arms. DXA performance data such as those in Fig. 2 enabled the novelties in CBMT to be licensed for commercialization (www.aeiouscientific.com).

\section{Validation}

Because bone strength can only be measured ex vivo, the accuracy of any method for estimating bone strength must be performed on cadaveric tissue. Figure 7 (left) shows the near identity $\left(\mathrm{R}^{2}=0.99\right)$ of ulna flexural rigidity $(\mathrm{EI})$ calculated from noninvasive CBMT and ex vivo quasistatic single load-to-failure (QMT) tests of ulna bending stiffness in 35 cadaveric human arms from male and female donors varying widely in age ( 17 to 99 years), height (1.47 to $1.88 \mathrm{~m}$; i.e., $4 \mathrm{ft}$. 10 in to $6 \mathrm{ft} .2 \mathrm{in})$, and body mass index (13 to $\left.40 \mathrm{~kg} / \mathrm{m}^{2}\right)$ [3•]. Figure 7 (right) shows the accuracy $\left(\mathrm{R}^{2}=0.99\right)$ of CBMT and QMT estimations of ulna bending strength $(\hat{Y})$ by ulna EI. The estimations were indistinguishable from one another $(p>0.80)$. Figure 7 (left) is evidence of the technical validity of CBMT (i.e., CBMT measures what it purports to measure), while Fig. 7 (right) is evidence of its clinical validity (i.e., CBMT measurements reflect the clinical condition of the patient: ulna bending strength).

\section{Total Error Rate}

Figure 7 (right) can be used to compare the total error rate (TER) of CBMT to that of other indirect methods for estimating direct QMT measures of bone strength for which corresponding graphs have been published. Table 1 compares TER values for estimations of ulna bending strength by CBMT measurements of ulna EI and by microtomographic measurements of cortical porosity [3•] as well as TER values for
Fig. 6 Wide angle (a) and close-up (b) views of a CBMT test in progress. The ulna is tested because, of all the bones in the body, the biomechanics of the ulna are the most ideal for a bending test in vivo.
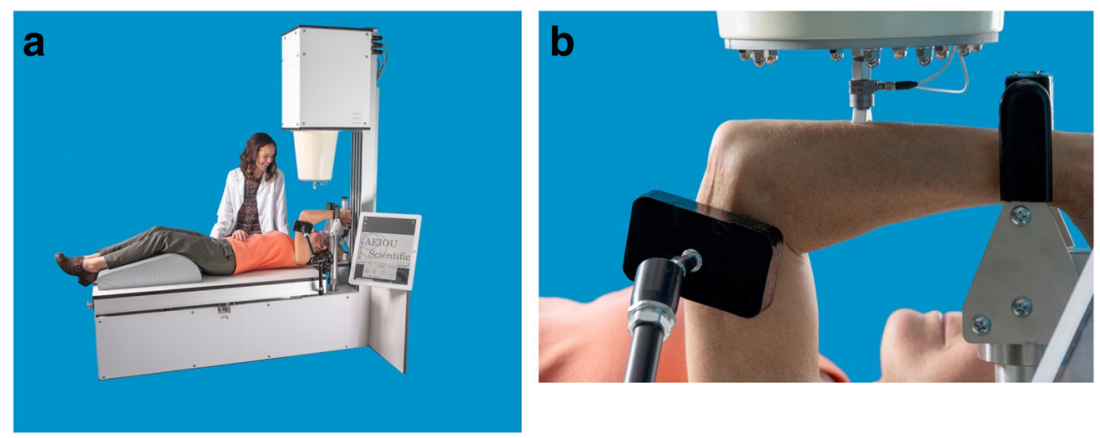
a

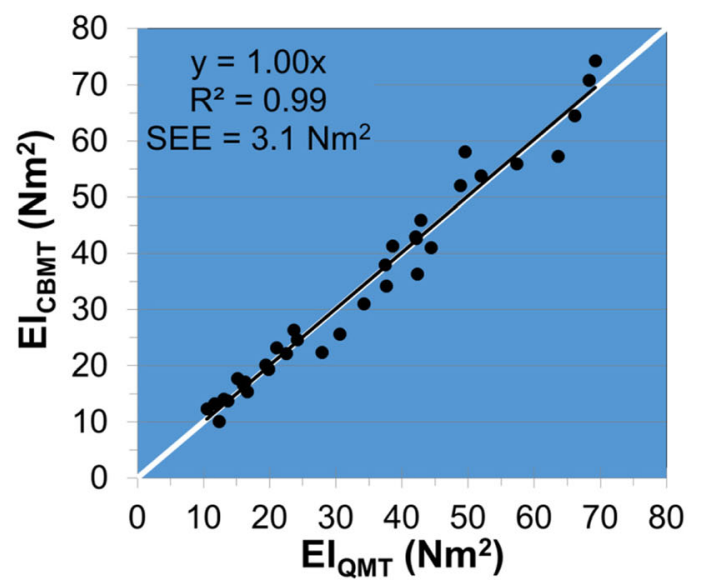

Fig. 7 CBMT technical validity (a): ulna flexural rigidity (EI) calculated from CBMT and quasistatic (QMT) measurements of ulna bending stiffness. CBMT clinical validity (b): ulna bending strength

estimations of femoral neck bending strength by femoral neck aBMD T-scores, tibial cortical porosity and tibial indentation (Biodent ${ }^{\mathrm{TM}}$; Active Life Sciences, Santa Barbara, CA) [23].

The high TER values for predictions of ulna and femoral neck bending strength by cortical porosity in Table 1 are consistent with high error rates $(\mathrm{FPR}=$ $75 \%, \mathrm{FNR}=67 \%$ ) in allocations of patients to treatment by cortical porosity [24]. Combining cortical porosity with aBMD T-scores improved but did not solve the problem $(\mathrm{FPR}=72 \%, \mathrm{FNR}=53 \%)$ [24].

Four models of reference point indentation devices have been produced over the years (Biodent ${ }^{\mathrm{TM}}$, Osteoprobe $\mathrm{I}^{\mathrm{TM}}$, Osteoprobe $\mathrm{II}^{\mathrm{TM}}$ and Osteoprobe ${ }^{\circledR}$; Active Life Sciences, Santa Barbara, CA), but the empirical basis of all of them is measurement of an increment of indentation by a stylus into the periosteal surface of a bone relative to one reference or another [25]. In general, indentation techniques measure hardness, which is not a mechanical property but rather a relationship between two materials. Differences in bone hardness have been detected between patients with and without various clinical conditions affecting bone health - sometimes [26-29], but the mechanical properties affecting indentation

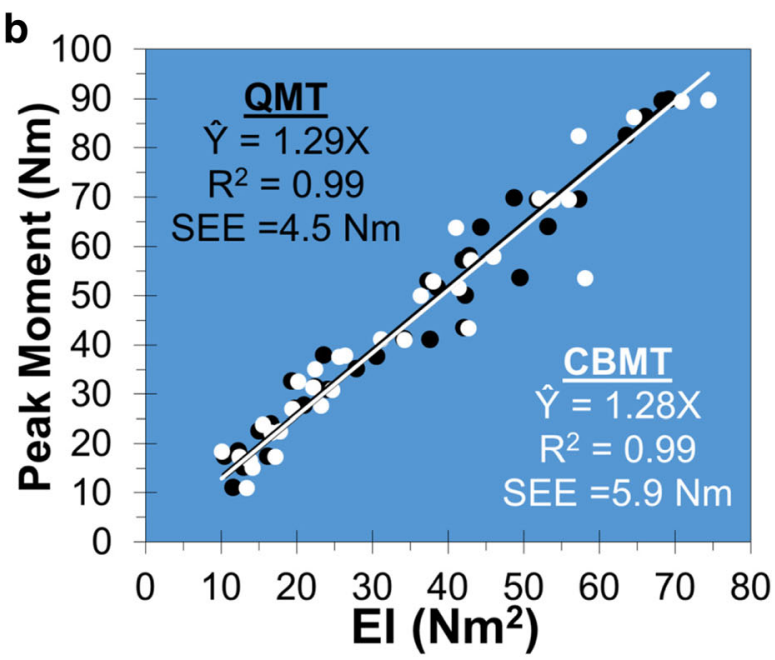

(peak moment) measured by QMT in excised ulnas in relation to ulna EI calculated from CBMT and QMT measurements of ulna bending stiffness. ( [3•], used with permission from Elsevier)

of bone are obscure [25, 30-32]. No significant association was found between indentation measured by the Osteoprobe ${ }^{\circledR}$ and any mechanical property of bone [32], and the strongest association reported between indentation measured by the Biodent ${ }^{\mathrm{TM}}$ and any mechanical property of bone $\left(\mathrm{R}^{2}=\right.$ 0.33) was with the toughness of hydrated cortical bone specimens $[31,33]$. As toughness is a material rather than structural mechanical property, the technique is now promoted for assessing cortical bone at the hierarchical level of bone tissue [34]. In contrast to indentation, the structural mechanical properties measured by CBMT (ulna stiffness, damping, and mass) are clear, and they characterize the mechanical behavior of a whole bone. The clinical utility of CBMT (i.e., whether its lower TER in predicting ulna bending strength improves the targeting of treatment at FN instead of FP and thereby patient outcomes) has not yet been tested.

\section{Dynamic Mechanical Loading}

When a person falls from a standing height, his hip hits the ground at up to 10 miles per hour. That is like running at a

Table 1 Total error rate (TER) for estimations of ulna and femoral neck bending strength indirectly by EI (flexural rigidity), aBMD T-scores ( $\mathrm{T}_{\mathrm{aBMD}}$ ), cortical porosity (CP), and indentation (IDI)

\begin{tabular}{|c|c|c|c|c|c|}
\hline Reference & Bone (n) & Predictor & SEE (Nm) & $\hat{Y}_{\max }-\hat{Y}_{\min }(\mathrm{Nm})$ & TER $(\%)$ \\
\hline \multirow[t]{2}{*}[3\bullet]{} & \multirow[t]{2}{*}{ Ulna (35) } & EI & 5.9 & 82.2 & $7 \%$ \\
\hline & & $\mathrm{CP}$ & $\begin{array}{l}18.9 \\
\operatorname{SEE}(\mathrm{N})\end{array}$ & $\begin{array}{l}55.8 \\
\hat{Y}_{\max }-\hat{Y}_{\min }(\mathrm{N})\end{array}$ & $34 \%$ \\
\hline \multirow[t]{3}{*}[23]{} & \multirow[t]{3}{*}{ Femoral neck (28) } & $\mathrm{T}_{\mathrm{aBMD}}$ & 1291 & 6476 & $20 \%$ \\
\hline & & Tibia CP & 1823 & 6146 & $44 \%$ \\
\hline & & Tibia IDI & 1837 & 4337 & $42 \%$ \\
\hline
\end{tabular}


$6 \mathrm{~min} / \mathrm{mile}$ pace - into a wall. Figure 8 shows stress-strain curves from single load-to-failure tests of bovine bone specimens under quasistatic (strain rate $\varepsilon^{\prime}=0.001 / \mathrm{s}$ ) and dynamic (i.e., higher strain rate) conditions. Note that the strength (i.e., peak stress before fracture) of bone tissue is much greater under dynamic loads than quasistatic ones. The following discussion explains why.

Under the dynamic conditions of a fall or collision, the relationship between force and motion in the linear elastic region of bone displacement is:

$\mathrm{F}=\mathrm{kx}+\mathrm{dv}+\mathrm{ma}$

where $\mathrm{F}$ is the force applied to the bone; where $\mathrm{x}, \mathrm{v}$, and a are resulting displacement, velocity, and acceleration of the bone; and where $\mathrm{k}, \mathrm{d}$, and $\mathrm{m}$ are bone's stiffness, damping, and mass. By contrast, under unrealistic, quasistatic conditions of a single load-to-failure test, $\mathrm{x}$ is made to increase so slowly that $\mathrm{v} \approx$ $\mathrm{a} \approx 0$ so that the relationship between force and motion reduces to:

$\mathrm{F}=\mathrm{kx}$

This enables the bone's stiffness to be measured as the slope of the force-displacement curve.

A bone can support much higher loads without breaking under dynamic conditions, because the higher loads are shared by the bone's viscous (dv) and inertial (ma) as well as elastic (kx) load carrying capacities. (The constant strain rates in Fig. 8 elicited only viscous and elastic effects.) Thus, bone damping and mass are protective against fracture under dynamic loads. Bone damping measured in our weakest

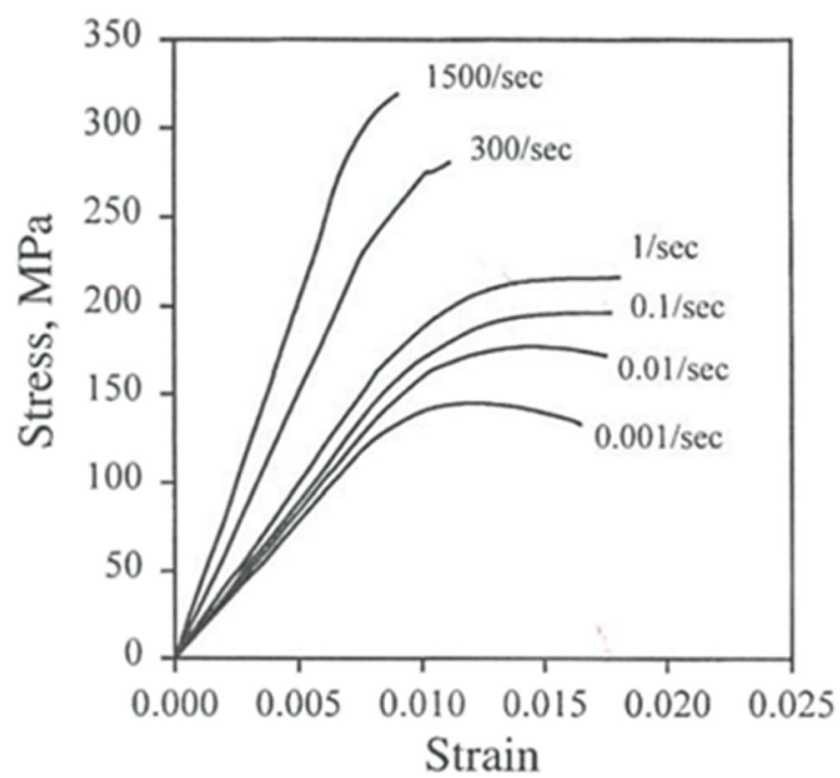

Fig. 8 Stress-strain curves for bovine bone specimens loaded at various constant strain rates (from [35] redrawn with permission from [36]). Used with permission from Springer Nature cadaveric ulnas would have increased their strength by $40 \%$ in a 5 mph collision.

Bone damping is a property of bone protein, and the elevated fracture risk in patients with Type 2 diabetes is attributed to the decoration of collagen with advanced glycation end products (AGEs) [37]. In our study of cadaveric arms, CBMT measurements of ulna damping were $25 \%$ lower in ulnas of nine donors with Type 2 diabetes than in ulnas of the same size in arms of donors without diabetes $(p=0.03)$. The technical validity of CBMT measurements of ulna damping and mass has not yet been confirmed by independent measures in $d y$ namic single load-to-failure tests. Nor has the clinical validity of those measurements been demonstrated by accurate estimates of ulna bending strength under the dynamic conditions of a fall or collision. Whether such clinically validated estimates are clinically useful for improving FPR and FNR also remains to be investigated.

\section{Conclusion}

Direct measurement of the mechanical properties of cortical bone in vivo may prove to be clinically useful in the assessment and monitoring of bone fragility in many clinical conditions besides osteoporosis associated with aging (including chronic kidney disease, HIV, cancer, cystic fibrosis, rheumatoid arthritis, bariatric surgery, spinal cord injury, androgen deprivation, corticosteroid treatment, anorexia nervosa, and the female athlete triad) as well as other systemic bone diseases (including osteogenesis imperfecta, osteomalacia, Paget's disease, hypophosphatasia, and hyperparathyroidism) and constitutional thinness. The commercially available CBMT scientific instrument shown in Fig. 6 is intended for use by scientists in studies of cortical bone mechanics in such human subjects. Its technical and clinical validity under quasistatic conditions have been demonstrated. Its validity under dynamic conditions remains to be determined. The CBMT scientific instrument is not a medical device and is not intended for use by health-care professionals to diagnose, monitor, or treat disease. For that purpose, an investigational CBMT medical device is under development. Its clinical utility has yet to be tested.

Funding Information Studies reported in this paper were funded by Ohio University and by the National Institute on Aging (1R43AG058312-01).

\section{Compliance with Ethical Standards}

Conflict of Interest Lyn Bowman is an investor on related US $(10,299,719)$ and European $(2,983,592)$ patents assigned to Ohio University and Licensed to AEIOU Scientific, LLC. Lyn Bowman and Anne Loucks are unit holders in AEIOU Scientific, LLC. 
Open Access This article is licensed under a Creative Commons Attribution 4.0 International License, which permits use, sharing, adaptation, distribution and reproduction in any medium or format, as long as you give appropriate credit to the original author(s) and the source, provide a link to the Creative Commons licence, and indicate if changes were made. The images or other third party material in this article are included in the article's Creative Commons licence, unless indicated otherwise in a credit line to the material. If material is not included in the article's Creative Commons licence and your intended use is not permitted by statutory regulation or exceeds the permitted use, you will need to obtain permission directly from the copyright holder. To view a copy of this licence, visit http://creativecommons.org/licenses/by/4.0/.

\section{References}

Papers of particular interest, published recently, have been highlighted as:

- Of importance

1. Borders S, Petersen KR, Orne D. Prediction of bending strength of long bones from measurements of bending stiffness and bone mineral content. J Biomech Eng. 1977;99(1):40-4. https://doi.org/10. $1115 / 1.3426267$

2. Jurist JM, Foltz AS. Human ulnar bending stiffness, mineral content, geometry and strength. J Biomech. 1977;10(8):455-9. https:// doi.org/10.1016/0021-9290(77)90099-9.

3. Bowman L, Ellerbrock ER, Hausfeld GC, Neumeyer JM, Loucks $\mathrm{AB}$. A new noninvasive mechanical bending test accurately predicts ulna bending strength in cadaveric human arms. Bone. 2019;120(3):336-46. https://doi.org/10.1016/j.bone.2018.11.018. A A detailed description of the technical and clinical validation of CBMT.

4. Bailey S, Vashishth D. Mechanical characterization of bone: state of the art in experimental approaches - what types of experiments do people do and how does one interpret the results? Curr Osteoporos Rep. 2018;16(4):423-33. https://doi.org/10.1007/s11914-0180454-8.

5. Black DM, Cauley JA, Wagman R, Ensrud K, Fink HA, Hillier TA, et al. The ability of a single BMD and fracture history assessment to predict fracture over 25 years in postmenopausal women: the study of osteoporotic fractures. J Bone Miner Res. 2018;33(3):389-95. https://doi.org/10.1002/jbmr.3194.

6. Nguyen TV, Eisman JA. Assessment of fracture risk: population association versus individual prediction. J Bone Miner Res. 2018;33(3):386-8. https://doi.org/10.1002/jbmr.3367.

7. Powers DMW. Evaluation: from precision, recall and F-measure to ROC, Informedness Markedness \& Correlation. J Mach Learn Tech. 2011;2(1):37-63.

8. Samelson EJ, Broe KE, Xu H, Yang L, Boyd S, Biver E, et al. Cortical and trabecular bone microarchitecture as an independent predictor of incident fracture risk in older women and men in the bone microarchitecture international consortium (BoMIC): a prospective study. Lancet Diabetes Endocrinol. 2019;7(1):34-43. https://doi.org/10.1016/S2213-8587(18)30308-5 A meta-analysis of eight studies of expected and actual fractures based on aBMD T-scores.

9. Wong AKO. Personal communication. September 5, 2019.

10. Biver E. Personal communication. September 13, 2019.

11. Boschitsch EP, Durchschlag E, Dimai HP. Age-related prevalence of osteoporosis and fragility fractures: real-world data from an Austrian menopause and osteoporosis clinic. Climacteric.
2017;20(2):157-63. https://doi.org/10.1080/13697137.2017. 1282452.

12. Lespessailles E, Cortet B, Legrand E, Guggenbuhl P, Roux C. Lowtrauma fractures without osteoporosis. Osteoporos Int. 2017;28(6): 1771-8. https://doi.org/10.1007/s00198-017-3921-7.

13. Nobakhti S, Shefelbine SJ. On the relation of bone mineral density and the elastic modulus in healthy and pathologic bone. Curr Osteoporos Rep. 2018;16(4):404-10. https://doi.org/10.1007/ s11914-018-0449-5.

14. Hernandez CJ, van der Meulen MC. Understanding bone strength is not enough. J Bone Miner Res. 2017;32(6):1157-62. https://doi. org/10.1002/jbmr.3078.

15. Hausfeld GC. Comparison of cortical porosity, diameter, and stiffness as predictors of ulna bending strength. Ohio University: OhioLINK Electronic Theses and Dissertations Center (Retrieved from https://etd.ohiolink.edu/); 2015.

16. Ramchand SK, Seeman E. The influence of cortical porosity on the strength of bone during growth and advancing age. Curr Osteoporos Rep. 2018;16(5):561-72. https://doi.org/10.1007/ s11914-018-0478-0.

17. Jarvinen TL, Michaelsson K, Aspenberg P, Sievanen H. Osteoporosis: the emperor has no clothes. J Intern Med. 2015;277(6):662-73. https://doi.org/10.1111/joim.12366.

18. Phelan EA, Mahoney JE, Voit JC, Stevens JA. Assessment and management of fall risk in primary care settings. Med Clin North Am. 2015;99(2):281-93. https://doi.org/10.1016/j.mcna.2014.11. 004.

19. Zebaze R, Seeman E. Cortical bone: a challenging geography. J Bone Miner Res. 2015;30(1):24-9. https://doi.org/10.1002/jbmr. 2419.

20. Steele CR, Zhou LJ, Guido D, Marcus R, Heinrichs WL, Cheema C. Noninvasive determination of ulnar stiffness from mechanical response - in vivo comparison of stiffness and bone mineral content in humans. J Biomech Eng. 1988;110(2):87-96. https://doi.org/ 10.1115/1.3108423.

21. Arnold PA, Ellerbrock ER, Bowman L, Loucks AB. Accuracy and reproducibility of bending stiffness measurements by mechanical response tissue analysis in artificial human ulnas. J Biomech. 2014;47(14):3580-3. https://doi.org/10.1016/j.jbiomech.2014.09. 005 .

22. Bowman L, Loucks AB. Improvements to mechanical response tissue analysis. MethodsX. 2019;6:2408-19 A description of improvements to Mechanical Response Tissue Analysis incorporated in Cortical Bone Mechanics Technology.

23. Abraham AC, Agarwalla A, Yadavalli A, McAndrew C, Liu JY, Tang SY. Multiscale predictors of femoral neck in situ strength in aging women: contributions of BMD, cortical porosity, reference point indentation, and nonenzymatic glycation. J Bone Miner Res. 2015;30(12):2207-14. https://doi.org/10.1002/jbmr.2568.

24. Boutroy S, Zebaze R, Sornay-Rendu E, Seeman E, Chapurlat R. Bone microstructure identifies women without osteoporosis suffering fragility fractures: the prospective OFELY study. ASBMR Annual Meeting; October 9-12, 2015; Seattle, WA2015.

25. Idkaidek A, Jasiuk I. Modeling of Osteoprobe indentation on bone. J Mech Behav Biomed Mater. 2019;90:365-73. https://doi.org/10. 1016/j.jmbbm.2018.09.037.

26. Farr JN, Drake MT, Amin S, Melton LJ 3rd, McCready LK, Khosla $\mathrm{S}$. In vivo assessment of bone quality in postmenopausal women with type 2 diabetes. J Bone Miner Res. 2014;29(4):787-95. https:// doi.org/10.1002/jbmr.2106

27. Nilsson AG, Sundh D, Johansson L, Nilsson M, Mellstrom D, Rudang R, et al. Type 2 diabetes mellitus is associated with better bone microarchitecture but lower bone material strength and poorer physical function in elderly women: a population-based study. J Bone Miner Res. 2017;32(5):1062-71. https://doi.org/10.1002/ jbmr.3057. 
28. Popp KL, Caksa S, Martinez-Betancourt A, Yuan A, Tsai J, Yu EW, et al. Cortical bone material strength index and bone microarchitecture in postmenopausal women with atypical femoral fractures. J Bone Miner Res. 2019;34(1):75-82. https://doi.org/10. 1002/jbmr.3590.

29. Rozental TD, Walley KC, Demissie S, Caksa S, MartinezBetancourt A, Parker AM, et al. Bone material strength index as measured by impact microindentation in postmenopausal women with distal radius and hip fractures. J Bone Miner Res. 2018;33(4): 621-6. https://doi.org/10.1002/jbmr.3338.

30. Allen MR, McNerny EM, Organ JM, Wallace JM. True gold or pyrite: a review of reference point indentation for assessing bone mechanical properties in vivo. J Bone Miner Res. 2015;30(9): 1539-50. https://doi.org/10.1002/jbmr.2603.

31. Krege JB, Aref MW, McNerny E, Wallace JM, Organ JM, Allen MR. Reference point indentation is insufficient for detecting alterations in traditional mechanical properties of bone under common experimental conditions. Bone. 2016;87:97-101. https://doi.org/10. 1016/j.bone.2016.04.002.

32. McNerny EM, Organ JM, Wallace JM, Newman CL, Brown DM, Allen MR. Assessing the inter- and intra-animal variability of in vivo OsteoProbe skeletal measures in untreated dogs. Bone Rep. 2016;5:192-8. https://doi.org/10.1016/j.bonr.2016.08.002.
33. Granke M, Coulmier A, Uppuganti S, Gaddy JA, Does MD, Nyman JS. Insights into reference point indentation involving human cortical bone: sensitivity to tissue anisotropy and mechanical behavior. J Mech Behav Biomed. 2014;37:174-85. https://doi.org/ 10.1016/j.jmbbm.2014.05.016.

34. Diez-Perez A, Bouxsein ML, Eriksen EF, Khosla S, Nyman JS, Papapoulos S, et al. Technical note: recommendations for a standard procedure to assess cortical bone at the tissue-level in vivo using impact microindentation. Bone Rep. 2016;5:181-5. https:// doi.org/10.1016/j.bonr.2016.07.004.

35. Martin RB, Burr DB, Sharkey NA. Mechanical Properties of Bone. Skeletal Tissue Mechanics. New York: Springer-Verlag New York, Inc; 1998. p. 127-80.

36. McElhaney JH. Dynamic response of bone and muscle tissue. $\mathrm{J}$ Appl Physiol. 1966;21(4):1231-6.

37. Acevedo C, Sylvia M, Schaible E, Graham JL, Stanhope KL, Metz $\mathrm{LN}$, et al. Contributions of material properties and structure to increased bone fragility for a given bone mass in the UCD-T2DM rat model of type 2 diabetes. J Bone Miner Res. 2018;33(6):1066-75. https://doi.org/10.1002/jbmr.3393.

Publisher's Note Springer Nature remains neutral with regard to jurisdictional claims in published maps and institutional affiliations. 